\title{
Prevalence of Nontuberculous Mycobacteria in a Tertiary Hospital in Beijing, China, January 2013 to December 2018
}

\author{
Jing-jing HUANG \\ Peking Union Medical College Hospital \\ Ying-xing LI \\ Peking Union Medical College Hospital \\ Ying ZHAO \\ Peking Union Medical College Hospital \\ Wen-hang YANG \\ Peking Union Medical College Hospital \\ Meng XIAO \\ Peking Union Medical College Hospital \\ Timothy KUDINHA \\ Charles Sturt University \\ Ying-chun XU ( $\nabla$ xycpumch@139.com )
}

Research article

Keywords: Mycobacterium; nontuberculous mycobacteria; identification; Gene chip

Posted Date: April 6th, 2020

DOI: https://doi.org/10.21203/rs.2.18053/v2

License: (c) (i) This work is licensed under a Creative Commons Attribution 4.0 International License. Read Full License

Version of Record: A version of this preprint was published at BMC Microbiology on June 12th, 2020. See the published version at https://doi.org/10.1186/s12866-020-01840-5. 


\section{Abstract}

Background To investigate the species distribution of non-tuberculous mycobacteria (NTM) among tuberculosis (TB) specimens collected from January 2013 to December 2018 at Peking Union Medical Hospital (Beijing), China. NTM species identification was carried out by DNA microarray chip. Results Mycobacterial species were detected in 1514 specimens from 1508 patients, among which NTM accounted for 37.3\% (565/1514), increasing from a proportion of $15.6 \%$ in 2013 to $46.1 \%$ in 2018 ( $P<0.001$ ). Among the 565 NTM positive specimens, the majority $(55.2 \%)$ were from female patients. Furthermore, patients aged $45-65$ years accounted for $49.6 \%$ of the total patients tested. Among 223 NTM positive specimens characterized further, the majority (86.2\%) were from respiratory tract, whilst $3.6 \%$ and $3.1 \%$ were from lymph nodes and pus, respectively. Mycobacterium intracellulare (31.8\%) and Mycobacterium chelonae / Mycobacterium abscessus (21.5\%) were the most frequently detected species, followed by M. avium (13.5\%), M. gordonae (11.7\%), M. kansasii (7.6\%), and others. Conclusion The proportion of NTM among mycobacterial species detected in a tertiary hospital in Beijing, China, increased rapidly from year 2013 to 2018. Middle-aged patients are more likely to be infected with NTM, especially females. Mycobacterium intracellulare and Mycobacterium chelonae / Mycobacterium abscessus were the most frequently detected NTM pathogens. Accurate and timely identification of NTM is important for diagnosis and treatment.

\section{Background}

The substantial increase in the number of patients with immunodeficiency disease in recent years has contributed to the rise in infectious diseases caused by a variety of rare organisms, including non-tuberculous mycobacteria (NTM) (1, 2). In China, patients diagnosed with or suspected of having tuberculosis (TB), are referred to a thoracic specialist hospital for further treatment. The clinical manifestations of TB and some NTM species infections are similar (3), with a considerable number of patients with putative NTM infections lost to follow up in tertiary hospitals. In addition, the treatment strategies used for management of infections caused by various NTM species are significantly different from those of TB (4). Thus accurate identification of Mycobacteria strains to species level is crucial for managing infections (5).

The distribution of NTM species in different clinical settings has been investigated by chest and/or TB hospitals in some provinces and regions of China (6, 7). However, data on these organisms from tertiary hospitals in China is limited. In 2013, the Non-tuberculous Mycobacteria Network European Trials Group (NTM-NET) reported on the geographic diversity of NTM species isolated from pulmonary samples, using data on isolates collected from 30 countries across six continents (8). The group found out that Mycobacterium avium complex (MAC) was the most frequently isolated species in most countries (e.g. Australia, Japan, and 13 countries in Europe), followed by M. gordonae and M. xenopi. However, this large study was not able to collect data from China.

Since 2013 to date, the Microbiology lab at Peking Union Medical College hospital has been using PCR-fluorescence probe method (Tuberculosis and NonTuberculous Mycobacteria Real-time PCR Detection Kit, CapitalBio Technology Inc., Beijing, China) to directly detect tuberculous/non-tuberculous Mycobacterium in clinical samples. DNA microarray chip method (Mycobacterial Species Identification Array Kit, CapitalBio Technology Inc., Beijing, China) was used to identify the different NTM species. These detections are crucial in the early diagnosis of patients with NTM infections. This study retrospectively analyzed the identification data of NTM samples from 2013 to 2018, to provide a general outline of the prevalence of non-tuberculous mycobacteria in a tertiary hospital in China.

\section{Methods}

\section{Data collection}

This was a retrospective study. Clinical data of patients who tested positive/negative for Mycobacterium tuberculosis (MTB) or NTM using a screening test (Real-time fluorescent PCR detection) from January 2013 to December 2018 in Peking Union Medical College Hospital, Beijing, China, were retrospectively collected. The study was approved by the Human Research Ethics Committee of Peking Union Medical College Hospital (no. S-K890). Data on Mycobacteria species identification, which was carried out by DNA microarray chip, was also collected. To avoid bias in our retrospective analysis, only one specimen per body site of an individual was included in the current study.

\section{PCR-fluorescence probe method}

\section{Nucleic acid extraction}

Tuberculosis and Non-Tuberculous Mycobacteria Real-time PCR Detection Kit (CapitalBio Technology Inc., Beijing, China) were used to extract nucleic acid from the specimens directly and thereafter Real-time fluorescent PCR for MTB or NTM screening was performed following the manufacturer's instructions. Nucleic acid extraction procedures were adapted according to the methodology described elsewhere (9). Briefly, the mixture of $4 \% \mathrm{NaOH}$ solution and the specimen (1:1) was vortexed and then incubated at room temperature for $30 \mathrm{~min}$. A $1 \mathrm{~mL}$ aliquot of this suspension was centrifuged at $12000 \mathrm{rpm}$ for $5 \mathrm{~min}$. The supernatant was discarded and $50 \mu \mathrm{L}$ of nucleic acid extract fluid was added to the pellet, vortexed thoroughly, and then put into Extractor ${ }^{\mathrm{TM}} 36 \mathrm{Nucleic}$ Acid Extractor (CapitalBio Technology Inc., Beijing, China) and centrifuged at maximum rotational speed for 5 min. The tube was subsequently put in a $95^{\circ} \mathrm{C}$ metal bath for $5 \mathrm{~min}$, centrifuged at $5000 \mathrm{rpm}$ for $1 \mathrm{~min}$, and then the supernatant could be preserved at $(-20 \pm 5){ }^{\circ} \mathrm{C}$ for one month.

\section{Real-time fluorescent PCR detection}

PCR amplification system was performed according to the manufacturer's instructions of Tuberculosis and Non-Tuberculous Mycobacteria Real-time PCR Detection Kit (CapitalBio Technology Inc., Beijing, China) (10) and was roughly as follows; $18 \mu \mathrm{L}$ PCR amplification reagent and $2 \mu \mathrm{L}$ template DNA were mixed together; amplification conditions: $37^{\circ} \mathrm{C}$ for $5 \mathrm{~min}, 94^{\circ} \mathrm{C}$ for $3 \mathrm{~min}$, and followed by the amplification of 40 cycles of $94{ }^{\circ} \mathrm{C}$ for $15 \mathrm{~s}, 60{ }^{\circ} \mathrm{C}$ for $30 \mathrm{~s}$, then $50{ }^{\circ} \mathrm{C}$ 
for $10 \mathrm{~s}$. FAM and HEX channels were selected for fluorescence detection at the same time. $60^{\circ} \mathrm{C}$ for 30 seconds was the fluorescence signal acquisition point. A result with a Ct value of $<40$ was considered positive. Negative and positive quality control products were included in parallel with the samples. Interpretation of real-time fluorescent PCR assay results is shown in Table 1.

\section{DNA microarray chip method}

DNA microarray chip from Mycobacterial Species Identification Array Kit (CapitalBio Technology Inc., Beijing, China) was performed for NTM species identification as previously reported $(11,12)$. Briefly, $9 \mu \mathrm{L}$ hybridization buffer and $6 \mu \mathrm{L} \mathrm{PCR}$ products were mixed; reaction conditions: $95^{\circ} \mathrm{C}$ for 5 min, ice bath for $3 \mathrm{~min}$, then blowing and mixing. After this $13.5 \mu \mathrm{L}$ of hybridization reaction mixture was added into the sampling hole, and then the hybridization box was sealed to maintain a $50{ }^{\circ} \mathrm{C}$ constant temperature in the water bath pot for $2 \mathrm{~h}$. Then washing and drying of the chip followed, and then scanning with LuxScan 10K-B Microarray Scanner (CapitalBio Technology Inc., Beijing, China). The corresponding software was used to read the signals and display the results.

\section{Statistical analyses}

Excel was used to establish the database, and SPSS 22.0 was used for statistical analysis. Trend analysis of annual constituent ratio was carried out by trend Chi-square tests and a $\mathrm{P}$ value $<0.01$ was considered statistically significant.

\section{Results}

\section{Changes of MTB/NTM detection rates}

From 2013 to 2018, 17287 non-repeat clinical specimens from Peking Union Medical College Hospital were sent to the Microbiology lab for detection of MTB/NTM by PCR-fluorescence probe method (Tuberculosis and Non-Tuberculous Mycobacteria Real-time PCR Detection Kit, CapitalBio Technology Inc., Beijing, China). Of these, 1514 (8.76\%) specimens were positive for MTB/NTM (Figure 1). During the six year study period, there was a significant increase in the number of samples tested for TB and/or NTM each year, with a gradual decrease in MTB detection, and a corresponding significant rise in NTM detection, year by year $\left(c^{2}=21.77 \otimes P<0.001\right)$, as shown in Figure 1. Thus altogether there were 1514 positive specimens obtained from 1508 patients, and 6 patients had mixed infection of MTB and NTM. MTB was detected in 949 (62.7\%) of the 1514 positive samples, and NTM in 565 (37.3\%). From 2013 to 2018 , there was a significant rise in the proportion of NTM, and a corresponding decrease in the proportion of MTB $\left(\mathrm{c}^{2}=58.84 \rrbracket P<0.001\right)$.

\section{Demographic data of NTM positive patients}

The 565 NTM positive patients comprised of $44.8 \%$ (253/565) males and $55.2 \%$ (312/565) females. The ages of the patients ranged from 10 to $95 y e a r s$, with an average age of $51.55 \pm 16.84$ years, and the quartiles were 40,53 and 63 years, respectively. Only 17 patients (17/565, 3.0\%) were under 18 years old. Patients aged $18-44$ years accounted for $28.3 \%$ (160/565) of the patients, those aged $45-65$ years for $49.6 \%(280 / 565)$, and those over 65 years of age accounted for $19.1 \%(108 / 565)$ of the total patients.

\section{Types of NTM positive specimens}

The 565 NTM positive samples were collected from different clinical departments. Most of them $(348 / 565,61.6 \%)$ were from the respiratory department, followed by infection department (55/565, 9.7\%), and other departments were relatively less represented (no one was more than $4.4 \%$ ). According to sample type category, the majority was sputum $(243 / 565,43.0 \%)$, followed by broncho-alveolar lavage fluid $(101 / 565,17.9 \%)$ and tracheobronchial aspiration $(74 / 565,13.1 \%)$, cerebrospinal fluid $(69 / 565,12.2 \%)$, lymph nodes $(19 / 565,3.4 \%)$, pus and urine (both $14 / 565,2.5 \%)$. Other types of specimens were less than $1.0 \%$. The total number of specimens from the respiratory tract was $418(74.0 \%)$.

\section{NTM species distribution}

Based on the requesting physician's requirements for further testing, 223 NTM positive samples were characterized for NTM species, with repetitive isolates from the same body part of the same patient excluded. These 223 samples were collected from 220 patients. Among them, two cases were multiple location infection, and one case was mixed infection of $M$. intracellulare and other Mycobacterium spp. isolate which was beyond the detection range of our kit and thus could not be identified to species level accurately. The NTM species identified by DNA microarray chip in the 223 samples included $M$. intracellulare (71), M. chelonae/M. abscessus (48), M. avium (30), M. gordonae (26), M. kansasii (17), M. fortuitum (15), M. xenopi (2), M. gilvum (2), M. smegmatis (1), M. marinum/M. ulcerans (1), M. terrae (1), M. phlei (1) and other Mycobacterium spp. (8). In addition, 7 specimens initially identified as other Mycobacterium spp. as they could not be identified to species level by Mycobacterial Species Identification Array Kit (CapitalBio Technology Inc., Beijing, China), were further identified as M. simiae (2), M. iranicum, M. chimaera, M. marswillense, M. holsaticum and M. colombiense by 16S rDNA sequencing. Distribution of NTM species identified by DNA microarray chip method during the period $2013-2018$, is shown in Table 2.

\section{NTM species distribution from different specimen types}

As shown in Table 3, there are several differences in sample sources for different NTM species. Besides the respiratory tract which was a major site for all NTM species, $45.5 \%(5 / 11)$ of $M$. chelonae/M. abscessus and 50.0\% (2/4) of $M$. kansasii were detected from lymph node specimens. Also, there were 33.3\% $(2 / 6)$ and $27.3 \%(3 / 11)$ of $M$. intracellulare and M. chelonae/M. abscessus detected from pus, respectively.

\section{Discussion}


Non-tuberculous mycobacteria (NTM) do not cause tuberculosis or leprosy, but can cause pulmonary disease resembling tuberculosis, lymphadenitis, skin disease, or disseminated disease. The pulmonary infection caused by NTM is difficult to clinically differentiate from that caused by MTB. However, most of the NTM strains are not susceptible to anti-tuberculosis drugs. The treatment plan is related to the species, infection site and the severity of infection (13). Therefore, rapid and accurate identification of Mycobacterium to species level is very important for managing these infections. PCR-fluorescence probe method (Tuberculosis and Non-Tuberculous Mycobacteria Real-time PCR Detection Kit, CapitalBio Technology Inc., Beijing, China) can be used to detect both MTB or NTM in the specimen directly in three hours, and there is no significant difference in the detection performance of this method compared to traditional methods (culture and microscopy) (10). In addition, the DNA microarray chip method (Mycobacterial Species Identification Array Kit, CapitalBio Technology Inc., Beijing, China) can detect and identify 17 species or groups of clinically common Mycobacteria, including M. tuberculosis complex, M. intracellular, M. avium, M. gordonae, M. kansasii, M. fortuitum, M. scrofulaceum, M. gilvum, M. terrae, M. chelonae /M. abscessus, M. phlei, M. nonchromogenicum, M. marinum /M. ulcerans, M. aureus, M. senegalense / M. malmoense, M. xenopi and M. smegmati. Using this method, results are available within 6 hours, which is helpful for early diagnosis and treatment of tuberculosis and NTM disease.

An analysis of the current study in relation to previous findings showed that the absolute numbers of both NTM and MTB positive samples increased yearly from 2013 to 2018. Notably, the proportion of NTM positive samples among the total positive samples for MTB and/or NTM as per the PCR-fluorescence probe method (Tuberculosis and Non-Tuberculous Mycobacteria Real-time PCR Detection Kit, CapitalBio Technology Inc., Beijing, China), increased from $15.6 \%$ in 2013 to $46.1 \%$ in 2018 . This is in agreement with the results of the national tuberculosis epidemiological sampling surveys carried out in China in 1990 (4.9\%), 2000 (11.1\%) and $2010(22.9 \%)(14,15)$.This finding is consistent with those from multiple studies in diverse countries, demonstrating an increasing proportion of NTM infections in recent years $(16,17)$. Many factors may contribute to the observed rise in NTM detection, including improved clinical awareness of NTM infections, use of better and more sensitive NTM detection techniques, increased number of immunocompromised patients, aging of the population, the threshold to test a sample, and alteration of sample types for NTM detection $(18,19)$. As shown in Figure 1 , as the number of samples submitted for MTB or NTM identification increased, so did the positivity rate for NTM species whilst that of MTB decreased. Besides, as shown in Table 4, the number of samples tested for NTM from cerebrospinal fluid (CSF) and lymph node has increased several times over the last three years. In addition, the NTM positive rate in CSF or lymph node sample is just lower than in sputum samples but higher than in any other samples. The increase in the numbers of CSF and lymph node samples tested may explain the observed increase in the number of NTM positive samples. Among the most common NTM pathogens, the incidence of MAC grew faster than that of M. chelonae /M. abscessus, from 2013 to 2018 (Table 2), suggesting a more prominent role for MAC in NTM infections. Furthermore, the fast increase in the proportion of M. gordona and M. fortuitum in NTM infections should be noted.

The major clinical manifestation of NTM is pulmonary disease, with MAC the most common species involved in infection (20, 21). In this retrospective study, M. intracellulare $(65 / 193,33.7 \%)$ was the most commonly detected species in respiratory tract samples, followed by $M$. chelonae / M. abscessus (37/193, 19.2\%), M. avium (27/193, 14.0\%), M. gordonae (24/193, 12.4\%). So in this study, MAC $(92 / 193,47.7 \%)$ was still the most common NTM pathogen in respiratory tract, which is consistent with previous findings in Europe, US, Canada, Australia, Japan, Korea, Southern China and so on (18-23). Although MAC is the most common pathogen in NTM pulmonary disease, its relative frequency varies widely among different geographical regions. Many factors such as climate type and population density can affect the distribution of NTM species (2).

In the present study, NTM infection was much more common in women (55.2\%) than men (44.8\%). Furthermore, the age range of infected people was relatively wide, being most common in the $45-65$ year age group (49.6\%), probably due to some issues related to the function of the immune system (24). This finding is in agreement with those of multiple studies in US $(25)$, Japan $(26,27)$ and South Korea $(23)$, which all indicated that older women were more susceptible to NTM infection. According to a previous study, abnormal expression of adipokines, sex hormones, and/or TGF- $\beta$ may predispose slender, older women to NTM infection (28). However, contrasting findings have been reported in Europe, where patients with NTM pulmonary disease were more likely to be male, possibly owing to smoking history and increased incidence of chronic obstructive pulmonary disease (COPD) (16, 27).

DNA microarray chip method (Mycobacterial Species Identification Array Kit, CapitalBio Technology Inc., Beijing, China) can accurately distinguish between M. avium and $M$. intracellulae, which have quite similar phenotypes. We found out that the prevalence of $M$. intracellulae (31.8\%) was always higher than $M$. avium (13.5\%) from 2013 to 2018. It is important to distinguish between M. avium and M. intracellulae because they show different pathogenic characteristics. M. intracellulae is more virulent compared to $M$. avium, indicating the need for a more intensive therapeutic strategy (29). However, the DNA microarray chip method (Mycobacterial Species Identification Array Kit, CapitalBio Technology Inc., Beijing, China) could not distinguish between M. chelonae and $M$. abscessus, and between M. marinum and M. ulcerans. This is because M. chelonae and $M$. abscessus have the same 16S rRNA gene sequences, and so are $M$. marinum and $M$. ulcerans. However, $M$. chelonae tends to cause disseminated infections (13), and $M$. ulcerans produces a cytotoxin (mycolactone) with immune-modulating properties that causes necrosis (30). Therefore, accurate identification of these strains to species level still has an important clinical significance. Microbiology laboratories could further identify these strains by 16S-23S Internal Transcribed Spacer Region sequencing and Sequencer-Based Capillary Gel Electrophoresis (31). Moreover, Mycobacterial Interspersed Repetitive Unit-Variable Number Tandem Repeat (MIRU-VNTR) markers can be used for typing $M$. intracellulae clinical isolates for molecular epidemiological studies (32). Other than pulmonary infectious diseases, NTM can also cause lymph node and skin and soft tissue infections. In this study, lymph node and pus were the second most common specimen types, only less than respiratory tract infections.

This study has several limitations. First, most NTM strains are widely distributed in the environment, being found in the soil, and water, including even treated water. Therefore, caution must be exercised in interpreting positive results from specimens as this doesn't necessarily mean that the patient is infected by the bacteria. Positive results may be due to bacterial colonization, or transient infection which is quickly cleared by the immune system, or contamination in sample collection and transportation (21). Second, the resolution power of our NTM detection method is limited. The Mycobacterial Species Identification Array Kit (CapitalBio Technology Inc., Beijing, China) cannot differentiate the following organisms into species level: $M$. chelonae and $M$. abscessus, $M$. marinum and M. ulcerans, M. szulgai and M. malmoense (11). In addition, the kit cannot discriminate NTM strains into sub-species level. Over recent years, researchers have modified the sub-species taxonomies of many NTM species $(33,34)$. The sub-species of $M$. abscessus, for instance, contains $M$. 
massiliense and $M$. bolletii, which are distinct in susceptibility to macrolides based on differences in the erm gene, thus leading to different therapeutic strategies if identified properly (34). Third, among all the NTM-positive samples collected (565), only $40 \%$ (223) were further subjected to species identification testing. This was because some patients were lost to follow up, and some patients could not afford to pay for further NTM species identification. As a result, our data may not contain the overall NTM species information.

Finally, among the common NTM species, MAC, M. abscessus and $M$. kansasii are highly pathogenic, and can cause lung diseases and lymph node, skin and soft tissue infections. However, M. gordonae and $M$. fortuitum rarely cause any infections, and only cause diseases when the patient's general condition is particularly poor. Thus during the clinical diagnosis and treatment, the clinical significance of the strain should be considered by combining with the patient's symptoms, signs and imaging findings.

\section{Conclusions}

The proportion of NTM strains among mycobacterial species detected in a tertiary hospital in Beijing, China, increased rapidly from year 2013 to 2018. Middle-aged and elderly patients are more likely to be infected with NTM, especially females. The most frequently detected NTM pathogens were $M$. intracellulare and M. chelonae / M. abscessus. Accurate and timely identification of NTM is crucial for diagnosis and treatment.

\section{Abbreviations}

MTB $₫$ Mcobacterium tuberculosis

NTM囚non-tuberculous mycobacteria

\section{Declarations}

\section{Ethics approval and consent to participate}

The retrospective study was approved by the Human Research Ethics Committee of Peking Union Medical College Hospital (no. S-K890).

\section{Consent for publication}

Not applicable

\section{Availability of data and materials}

The datasets analyzed during the current study are not publicly available due to the privacy of patients but are available from the corresponding author on reasonable request.

\section{Competing interests}

The authors declare that they have no competing interests

\section{Funding}

This work was supported by the National Major Science and Technology Projects for the Control and Prevention of Major Infectious Diseases of China (2017ZX10103004, 2018ZX10712001) and the Fundamental Research Funds for the Central Universities (3332018035, 3332018041).

The funding bodies had no role in study design, data collection and analysis, interpretation of data, decision to publish, or preparation of the manuscript.

\section{Authors' contributions}

$\mathrm{JJH}$ analyzed and interpreted the patient data. WHY collected the data from two systems of laboratory. MX and YCX guided the idea of analysis. YZ, YXL and TK were major contributors in writing the manuscript. All authors read and approved the final manuscript.

\section{Acknowledgements}

The authors are grateful to all the colleagues that participated in routine tests of Mycobacterium tuberculosis and non-tuberculous mycobacteria in department of clinical microbiology, Peking Union Medical College Hospital, Beijng, China.

\section{References}

1. Honda JR, Knight V, Chan ED. Pathogenesis and risk factors for nontuberculous mycobacterial lung disease. Clin Chest Med. 2015;36(1):1-11.

2. Zhang ZX, Cherng BPZ, Sng LH, Tan YE. Clinical and microbiological characteristics of non-tuberculous mycobacteria diseases in Singapore with a focus on pulmonary disease, 2012-2016. BMC Infect Dis. 2019;19(1):436.

3. Hu C, Huang L, Cai M, Wang W, Shi X, Chen W. Characterization of non-tuberculous mycobacterial pulmonary disease in Nanjing district of China. BMC Infect Dis. 2019;19(1):764.

4. Porvaznik I, Solovic I, Mokry J. Non-Tuberculous Mycobacteria: Classification, Diagnostics, and Therapy. Adv Exp Med Biol. 2017;944:19-25. 
5. Griffith DE, Aksamit T, Brown-Elliott BA, Catanzaro A, Daley C, Gordin F, et al. An official ATS/IDSA statement: diagnosis, treatment, and prevention of nontuberculous mycobacterial diseases. Am J Respir Crit Care Med. 2007;175(4):367-416.

6. Tan Y, Su B, Shu W, Cai X, Kuang S, Kuang H, et al. Epidemiology of pulmonary disease due to nontuberculous mycobacteria in Southern China, 20132016. BMC pulmonary medicine. 2018;18(1):168.

7. Liu H, Lian L, Jiang Y, Huang M, Tan Y, Zhao X, et al. Identification of Species of Nontuberculous Mycobacteria Clinical Isolates from 8 Provinces of China. BioMed research international. 2016;2016:2153910.

8. Hoefsloot W, van Ingen J, Andrejak C, Angeby K, Bauriaud R, Bemer P, et al. The geographic diversity of nontuberculous mycobacteria isolated from pulmonary samples: an NTM-NET collaborative study. Eur Respir J. 2013;42(6):1604-13.

9. Guo Y, Zhou Y, Wang C, Zhu L, Wang S, Li Q, et al. Rapid, accurate determination of multidrug resistance in M. tuberculosis isolates and sputum using a biochip system. The international journal of tuberculosis and lung disease : the official journal of the International Union against Tuberculosis and Lung Disease. 2009;13(7):914-20.

10. Guo L, Xu Y, Sun H, Song H, Wang Y, Zhao Y. [Clinical application of real-time FQ-PCR assay in rapid detection of Mycobacterium spp infection] (in Chinese). Chin J Nosocomio. 2015;25(21):4811-3.

11. Liu J, Yue J, Yan Z, Han M, Han Z, Jin L, et al. Performance assessment of the CapitalBio mycobacterium identification array system for identification of mycobacteria. J Clin Microbiol. 2012;50(1):76-80.

12. Shi XC, Liu XQ, Xie XL, Xu YC, Zhao ZX. Gene chip array for differentiation of mycobacterial species and detection of drug resistance. Chinese medical journal. 2012;125(18):3292-7.

13. Falkinham JO, 3rd. Environmental sources of nontuberculous mycobacteria. Clin Chest Med. 2015;36(1):35-41.

14. Technical Guidance Group of the Fifth National TB Epidemiological Survey. [The fifth national tuberculosis epidemiological survey in China in 2010] (in Chinese). Chin J Antituberc. 2012;34(08):485-508.

15. National Technical Steering Group of the Epidemiological Sampling Survey for Tuberculosis. [Report on nationwide random survey for the epidemiology of tuberculosis in 2000] (in Chinese). Chin J Antituberc. 2002(02):3-46.

16. Prevots DR, Loddenkemper R, Sotgiu G, Migliori GB. Nontuberculous mycobacterial pulmonary disease: an increasing burden with substantial costs. Eur Respir J. 2017;49(4).

17. Donohue MJ, Wymer L. Increasing Prevalence Rate of Nontuberculous Mycobacteria Infections in Five States, 2008-2013. Ann Am Thorac Soc. 2016;13(12):2143-50.

18. Horne D, Skerrett S. Recent advances in nontuberculous mycobacterial lung infections. F1000Res. 2019;8.

19. Namkoong H, Kurashima A, Morimoto K, Hoshino Y, Hasegawa N, Ato M, et al. Epidemiology of Pulmonary Nontuberculous Mycobacterial Disease, Japan. Emerg Infect Dis. 2016;22(6):1116-7.

20. Prevots DR, Marras TK. Epidemiology of human pulmonary infection with nontuberculous mycobacteria: a review. Clin Chest Med. 2015;36(1):13-34.

21. Stout JE, Koh WJ, Yew WW. Update on pulmonary disease due to non-tuberculous mycobacteria. Int J Infect Dis. 2016;45:123-34.

22. Brode SK, Marchand-Austin A, Jamieson FB, Marras TK. Pulmonary versus Nonpulmonary Nontuberculous Mycobacteria, Ontario, Canada. Emerg Infect Dis. 2017;23(11):1898-901.

23. Lee H, Myung W, Koh WJ, Moon SM, Jhun BW. Epidemiology of Nontuberculous Mycobacterial Infection, South Korea, 2007-2016. Emerg Infect Dis. 2019;25(3):569-72.

24. Marras TK, Mehta M, Chedore P, May K, Al Houqani M, Jamieson F. Nontuberculous mycobacterial lung infections in Ontario, Canada: clinical and microbiological characteristics. Lung. 2010;188(4):289-99.

25. Adjemian J, Olivier KN, Seitz AE, Holland SM, Prevots DR. Prevalence of nontuberculous mycobacterial lung disease in U.S. Medicare beneficiaries. Am J Respir Crit Care Med. 2012;185(8):881-6.

26. Morimoto K, Iwai K, Uchimura K, Okumura M, Yoshiyama T, Yoshimori K, et al. A steady increase in nontuberculous mycobacteriosis mortality and estimated prevalence in Japan. Ann Am Thorac Soc. 2014;11(1):1-8.

27. van Ingen J, Wagner D, Gallagher J, Morimoto K, Lange C, Haworth CS, et al. Poor adherence to management guidelines in nontuberculous mycobacterial pulmonary diseases. Eur Respir J. 2017;49(2).

28. Chan ED, Iseman MD. Slender, older women appear to be more susceptible to nontuberculous mycobacterial lung disease. Gend Med. 2010;7(1):5-18.

29. Jang MA, Koh WJ, Huh HJ, Kim SY, Jeon K, Ki CS, et al. Distribution of nontuberculous mycobacteria by multigene sequence-based typing and clinical significance of isolated strains. J Clin Microbiol. 2014;52(4):1207-12.

30. Gehringer M, Altmann KH. The chemistry and biology of mycolactones. Beilstein J Org Chem. 2017;13:1596-660.

31. Subedi S, Kong F, Jelfs P, Gray TJ, Xiao M, Sintchenko V, et al. 16S-23S Internal Transcribed Spacer Region PCR and Sequencer-Based Capillary Gel Electrophoresis has Potential as an Alternative to High Performance Liquid Chromatography for Identification of Slowly Growing Nontuberculous Mycobacteria. PLoS One. 2016;11(10):e0164138.

32. Dauchy FA, Degrange S, Charron A, Dupon M, Xin Y, Bebear C, et al. Variable-number tandem-repeat markers for typing Mycobacterium intracellulare strains isolated in humans. BMC Microbiol. 2010;10:93.

33. Jagielski T, Borowka P, Bakula Z, Lach J, Marciniak B, Brzostek A, et al. Genomic Insights Into the Mycobacterium kansasii Complex: An Update. Front Microbiol. 2019;10:2918. 
34. Tortoli E, Kohl TA, Brown-Elliott BA, Trovato A, Leao SC, Garcia MJ, et al. Emended description of Mycobacterium abscessus, Mycobacterium abscessus subsp. abscessus and Mycobacterium abscessus subsp. bolletii and designation of Mycobacterium abscessus subsp. massiliense comb. nov. Int J Syst Evol Microbiol. 2016;66(11):4471-9.

\section{Tables}

Table 1. Interpretation of real-time fluorescent PCR assay results

\begin{tabular}{ll}
\hline Detection results & Interpretation \\
\hline FAM (+) HEX(+) & MTB nucleic acid detection positive \\
FAM (+) HEX(-) & MTB nucleic acid detection positive \\
FAM (-) HEX (+) & NTM nucleic acid detection positive \\
FAM (-) HEX(-) & Mycobacterium spp. nucleic acid detection negative \\
\hline
\end{tabular}

MTB: Mycobacterium tuberculosis; NTM $\llbracket$ non-tuberculous mycobacteria

Table 2. Non-tuberculous species identified from 2013 to 2018 in a tertiary hospital in Beijing, China.

\begin{tabular}{lccccccc}
\hline $\begin{array}{l}\text { Gene chip identified } \\
\text { Mycobacterial Species }\end{array}$ & 2013 & 2014 & 2015 & 2016 & 2017 & 2018 & Total (\%) \\
\hline M. intracellulare & 2 & 2 & 8 & 8 & 20 & 31 & $71(31.8)$ \\
\hline M. chelonae/M. abscessus & 2 & 3 & 11 & 13 & 10 & 9 & $48(21.5)$ \\
\hline M. avium & 0 & 1 & 5 & 2 & 6 & 16 & $30(13.5)$ \\
\hline M. gordonae & 0 & 1 & 2 & 3 & 8 & 12 & $26(11.7)$ \\
\hline M. kansasii & 0 & 0 & 2 & 6 & 4 & 5 & $17(7.6)$ \\
\hline M. fortuitum & 0 & 0 & 1 & 2 & 4 & 8 & $15(6.7)$ \\
\hline M. gilvum & 0 & 0 & 0 & 0 & 1 & 1 & $2(0.9)$ \\
\hline M. xenopi & 0 & 0 & 0 & 0 & 1 & 1 & $2(0.9)$ \\
\hline M. marinum/M. ulcerans & 0 & 1 & 0 & 0 & 0 & 0 & $1(0.4)$ \\
\hline M. smegmati & 0 & 0 & 1 & 0 & 0 & 0 & $1(0.4)$ \\
\hline M. terrae & 0 & 0 & 0 & 0 & 0 & 1 & $1(0.4)$ \\
\hline M. phlei & 0 & 0 & 0 & 0 & 0 & 1 & $1(0.4)$ \\
\hline Other Mycobacterium & 0 & 0 & 0 & 2 & 1 & 5 & $8 *(3.6)$ \\
\hline Total & 4 & 8 & 30 & 36 & 55 & 90 & $223(100)$ \\
\hline
\end{tabular}

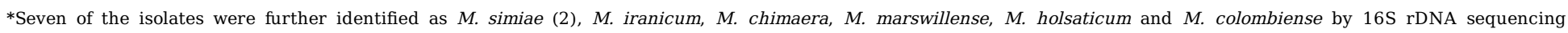
respectively.

Table 3. Specimen types among which NTM were identified in this study.

\begin{tabular}{|c|c|c|c|c|c|c|c|c|c|c|c|c|c|c|c|}
\hline $\begin{array}{l}\text { lentified } \\
\text { ial Species }\end{array}$ & Sputum & $\begin{array}{c}\text { Bronchoalveolar } \\
\text { lavage fluid }\end{array}$ & $\begin{array}{c}\text { Tracheobronchial } \\
\text { aspiration }\end{array}$ & $\begin{array}{l}\text { Lymph } \\
\text { node }\end{array}$ & Pus & Hydrothorax & Urine & $\begin{array}{l}\text { Lung } \\
\text { tissue }\end{array}$ & $\begin{array}{l}\text { Ascitic } \\
\text { fluid }\end{array}$ & Marrow & $\begin{array}{l}\text { Joint } \\
\text { fluid }\end{array}$ & $\begin{array}{c}\text { Subcutaneous } \\
\text { nodule }\end{array}$ & $\begin{array}{c}\text { Vertebrae } \\
\text { puncture } \\
\text { tissue }\end{array}$ & Others & Total \\
\hline ulare & 46 & 12 & 7 & 0 & 2 & 1 & 0 & 0 & 0 & 1 & 1 & 1 & 0 & 0 & 71 \\
\hline \multicolumn{16}{|l|}{$/ M$} \\
\hline & 35 & 1 & 1 & 5 & 3 & 1 & 0 & 0 & 0 & 0 & 0 & 0 & 0 & 2 & 48 \\
\hline & 18 & 8 & 1 & 1 & 0 & 1 & 0 & 1 & 0 & 0 & 0 & 0 & 0 & 0 & 30 \\
\hline \multirow[t]{2}{*}{3} & 20 & 4 & 0 & 0 & 0 & 1 & 1 & 0 & 0 & 0 & 0 & 0 & 0 & 0 & 26 \\
\hline & 9 & 4 & 0 & 2 & 1 & 0 & 0 & 0 & 0 & 0 & 0 & 0 & 1 & 0 & 17 \\
\hline \multirow[t]{3}{*}{1} & 13 & 1 & 0 & 0 & 0 & 0 & 1 & 0 & 0 & 0 & 0 & 0 & 0 & 0 & 15 \\
\hline & 2 & 0 & 0 & 0 & 0 & 0 & 0 & 0 & 0 & 0 & 0 & 0 & 0 & 0 & 2 \\
\hline & 2 & 0 & 0 & 0 & 0 & 0 & 0 & 0 & 0 & 0 & 0 & 0 & 0 & 0 & 2 \\
\hline \multicolumn{16}{|l|}{$/ M$} \\
\hline & 0 & 0 & 0 & 0 & 1 & 0 & 0 & 0 & 0 & 0 & 0 & 0 & 0 & 0 & 1 \\
\hline \multirow[t]{3}{*}{$i$} & 0 & 0 & 0 & 0 & 0 & 0 & 0 & 0 & 1 & 0 & 0 & 0 & 0 & 0 & 1 \\
\hline & 1 & 0 & 0 & 0 & 0 & 0 & 0 & 0 & 0 & 0 & 0 & 0 & 0 & 0 & 1 \\
\hline & 1 & 0 & 0 & 0 & 0 & 0 & 0 & 0 & 0 & 0 & 0 & 0 & 0 & 0 & 1 \\
\hline \multirow[t]{2}{*}{ bacterium } & 7 & 0 & 0 & 0 & 0 & 0 & 0 & 0 & 0 & 0 & 0 & 0 & 0 & 1 & 8 \\
\hline & 154 & 30 & 9 & 8 & 7 & 4 & 2 & 1 & 1 & 1 & 1 & 1 & 1 & 3 & 223 \\
\hline
\end{tabular}

Table 4. The numbers of each different sample type tested for mycobacteria. 


\begin{tabular}{lccccccc}
\hline \multirow{2}{*}{ Sample types } & \multicolumn{7}{c}{ No. of tested samples (\%) } \\
\cline { 2 - 7 } & 2013 & 2014 & 2015 & 2016 & 2017 & 2018 & Total \\
\hline Sputum & $402(46.8)$ & $626(33.7)$ & $954(42.0)$ & $1482(45.5)$ & $1940(47.0)$ & $2271(46.2)$ & $7675(44.4)$ \\
\hline Tracheobronchial aspiration & $160(18.6)$ & $524(28.2)$ & $570(25.1)$ & $587(18.0)$ & $765(18.5)$ & $793(16.1)$ & $3399(19.7)$ \\
\hline Cerebrospinal fluid & $57(6.6)$ & $109(5.9)$ & $155(6.8)$ & $358(11.0)$ & $452(10.9)$ & $697(14.2)$ & $1828(10.6)$ \\
\hline Bronchoalveolar lavage fluid & $76(8.8)$ & $209(11.3)$ & $184(8.1)$ & $216(6.6)$ & $292(7.1)$ & $323(6.6)$ & $1300(7.5)$ \\
\hline Hydrothorax & $57(6.6)$ & $90(4.8)$ & $116(5.1)$ & $175(5.4)$ & $194(4.7)$ & $207(4.2)$ & $839(4.9)$ \\
\hline Urine & $43(5.0)$ & $82(4.4)$ & $93(4.1)$ & $127(3.9)$ & $141(3.4)$ & $194(4.0)$ & $680(3.9)$ \\
\hline Lymph node & $3(0.3)$ & $36(1.9)$ & $32(1.4)$ & $128(3.9)$ & $104(2.5)$ & $149(3.0)$ & $452(2.6)$ \\
\hline Ascitic fluid & $16(1.9)$ & $56(3.0)$ & $46(2.0)$ & $85(2.6)$ & $81(2.0)$ & $92(1.9)$ & $376(2.2)$ \\
\hline Pus & $22(2.6)$ & $24(1.3)$ & $32(1.4)$ & $35(1.1)$ & $46(1.1)$ & $64(1.3)$ & $223(1.3)$ \\
\hline Others & $23(2.7)$ & $101(5.4)$ & $89(3.9)$ & $66(2.0)$ & $115(2.8)$ & $121(2.5)$ & $515(3.0)$ \\
\hline
\end{tabular}

\section{Figures}

\section{$=\mathrm{MTB}=0 \mathrm{NTM}$}

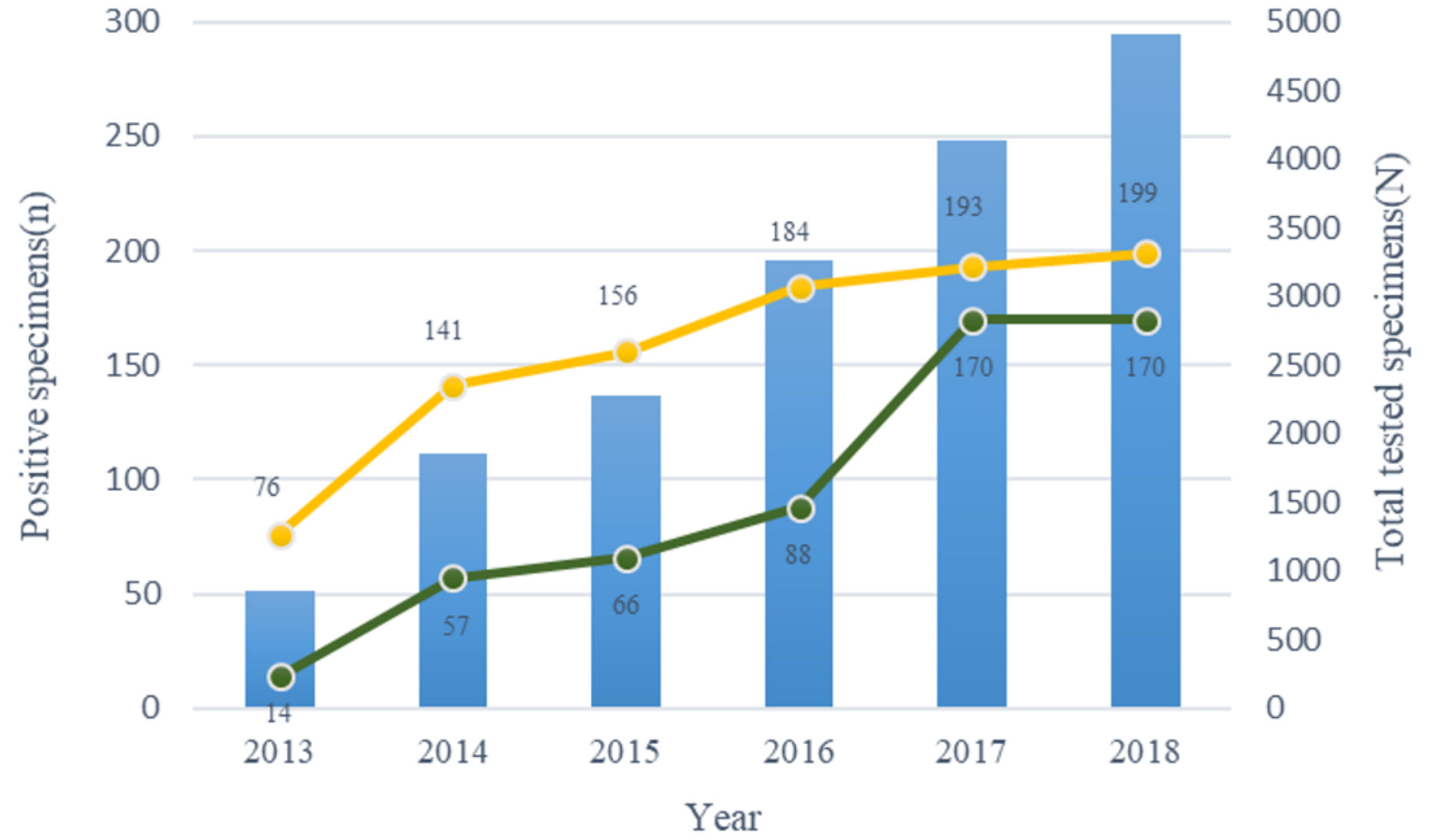

\section{Figure 1}

Distribution of Positive specimens in Mycobacterium nucleic acid detection during 2013 - 2018. MTB: Mycobacterium tuberculosis; NTM $\llbracket$ non-tuberculous mycobacteria 